\title{
Comparación de la eficacia del pentobarbital y el tiopental en el control de la hipertensión intracraneal refractaria. Resultados preliminares en una serie de $\mathbf{2 0}$
}

\section{pacientes}

J. Pérez-Bárcena; B. Barceló*; J. Homar; J.M. Abadal; F.J. Molina**; A. de la Peña***; J. Sahuquillo**** y J. Ibáñez

Servicio de Medicina Intensiva. Hospital Son Dureta. Palma Mallorca. Departament de Cirurgia. Universitat Autònoma. Barcelona. Servicios de Análisis Clínicos* y de Neurología**. Hospital Son Dureta. Palma Mallorca. Servicio de Medicina Interna***. Hospital Son LLatzer. Palma Mallorca. Servicio de Neurocirugía****. Hospital Vall d’Hebrón. Barcelona. Departament de Cirurgia. Universitat Autònoma. Barcelona.

\section{Resumen}

Objetivo. Comparar la eficacia del pentobarbital y tiopental en el control de la hipertensión intracraneal (HIC) refractaria a las medidas de primer nivel, según las Guías de Práctica Clínica de la "Brain Trauma Foundation", en pacientes con traumatismo craneoencefálico (TCE) grave.

Material y métodos. Estudio prospectivo de cohorte, aleatorizado, de intervención terapéutica comparativa entre dos fármacos: pentobarbital y tiopental. La muestra fue seleccionada a partir de los pacientes que sufrieron un TCE grave (Glasgow Coma Scale GCS, postresucitación, $\leq 8$ puntos o deterioro neurológico en la primera semana post traumatismo) y que presentaron HIC (PIC $>20 \mathrm{mmHg}$ ) refractaria al tratamiento convencional, de acuerdo con las Guías de Práctica Clínica de la "Brain Trauma Foundation". Además de comprobar la eficacia en el control de la PIC, también se recogieron los efectos secundarios del tratamiento.

Resultados. Se presentan los resultados de los primeros 20 pacientes reclutados, de los cuales diez recibieron tiopental y diez pentobarbital. No hubo diferencias estadísticamente significativas en las características basales de los pacientes (edad, sexo, índices de gravedad y comorbilidades). Tampoco hubo diferencias respecto al GCS de ingreso (tiopental seis puntos; pentobarbital siete puntos; $P=0,26$ ) ni en la tomografía craneal computarizada de ingreso, según la clasificación del "Traumatic Coma Data Bank".

En el grupo de tiopental se controló la PIC en cinco casos y en el grupo de pentobarbital en dos pacientes $(\mathrm{P}=\mathbf{0 , 1 6})$. Cinco pacientes tratados con tiopental fallecieron y ocho en el grupo de pentobarbital $(P=0,16)$.

No hubo diferencias significativas entre ambos grupos respecto a la incidencia de hipotensión arterial $(P=1)$ o complicaciones infecciosas.

Conclusiones. los resultados preliminares de este estudio indican que el tiopental podría ser más eficaz

Recibido: 25-05-04. Aceptado: 18-08-04 que el pentobarbital en el control de la HIC refractaria a las medidas de primer nivel. Dichos resultados confirman la evidencia experimental que indica que los mecanismos neuroprotectores de ambos fármacos son distintos y justifican el continuar aumentando el tamaño muestral de nuestro estudio para poder definir mejor la eficacia de ambos fármacos.

PALABRAS CLAVE: Traumatismo craneal. Hipertensión intracraneal. Pentobarbital. Tiopental.

Comparison of the effectiveness of pentobarbital and thiopental in patients with refractory intracranial hypertension. Preliminary report of 20 patients

Objective. To assess the effectiveness of pentobarbital and thiopental to control raised intracranial pressure (ICP), refractory to first level measures, in patients with severe traumatic brain injury.

Material and methods. Prospective, randomized study to compare the effectiveness between two treatments: pentobarbital and thiopental. The patients will be selected from those admitted to the Intensive Care Unit with a severe traumatic brain injury (postresuscitation Glasgow Coma Scale equal or less than 8 points) and raised ICP (ICP $>20 \mathrm{mmHg})$ refractory to first level measures according to the Brain Trauma Foundation guidelines. The adverse effects of both treatments were also collected.

Results. We present the results of the first 20 patients included. Ten received pentobarbital and the other ten thiopental. There were no statistically significance

Abreviaturas. APACHE: Acute Physiology and Chronic Health Evaluation. FSC: flujo sanguíneo cerebral. GCS: Glasgow Coma Scale. HIC: hipertensión intracraneal. ISS: injury severity score. LCR: líquido cefalorraquídeo. PIC: presión intracraneal. SAPS: simplified acute physiologym score. SNC: sistema nervioso central. TAM: tensión arterial media. TC: tomografia computarizada. TCE: traumatismo craneoencefálico grave. VSC: volumen sanguíneo central 
differences in patients'characteristics (age, sex, severity of the trauma at admission and comorbilities). There were no differences between both groups neither in the Glasgow Coma Scale at admission (thiopental six points; pentobarbital seven points; $P=0,26$ ) nor in the admission Cranial Tomography, according to the Traumatic Coma Data Bank classification.

Thiopental treatment controlled raised ICP in five cases and pentobarbital in two cases $(P=0,16)$. Five patients in the thiopental group died and eight in the pentobarbital group $(\mathrm{P}=\mathbf{0}, \mathbf{1 6})$.

There were no statistically differences between both groups regarding to the presence of hypotension $(\mathrm{P}=1)$ or infectious complications.

Conclusions. These preliminary results indicate that thiopental could be more effective than pentobarbital in patients with refractory intracranial hypertension. These results support previous experimental findings that show that both treatments are not equal and justify to continue this study.

KEY WORD: Head injury. Intracranial hypertension. Pentobarbital. Thiopental

\section{Introducción}

Los barbitúricos se han utilizado desde ha décadas en el tratamiento de los pacientes con un traumatismo craneoencefálico (TCE) grave ${ }^{13,15-17}$. Fue Horsley el primero en demostrar su eficacia en reducir la presión intracraneal (PIC $)^{10}$. Actualmente, el empleo de barbitúricos a altas dosis se utiliza en aquellos pacientes con un TCE grave que presentan hipertensión intracraneal (HIC) refractaria al tratamiento médico y quirúrgico. Esta indicación está recogida en las Guías de Práctica Clínica de la "Brain Trauma Foundation", siendo este proceder el único tratamiento de "segundo nivel" del que existe evidencia clase II para justificar su utilización ${ }^{2}$.

Los barbitúricos son fármacos derivados del ácido barbitúrico, que tienen en común el disminuir la excitabilidad de todas las células excitables del organismo, sobre todo en el sistema nervioso central (SNC). La acción más importante de los barbitúricos sobre el encéfalo está en relación con su capacidad para reducir el metabolismo cerebral y al efecto consecuente de que el metabolismo se encuentra acoplado al flujo sanguíneo cerebral (FSC). Al reducir su metabolismo, el encéfalo reduce de forma casi lineal el FSC y por lo tanto también disminuye el volumen sanguíneo cerebral (VSC). La reducción volumétrica del contenido sanguíneo se acompaña de una reducción de la PIC en aquellos pacientes con $\mathrm{HIC}^{5,12,20,21}$.

Los barbitúricos se han clasificado atendiendo a su vida media de eliminación y a su tiempo de acción. La clasi- ficación tradicional los agrupa en barbitúricos de acción ultracorta, corta, intermedia y larga. La farmacocinética y farmacodinamia de los barbitúricos está en relación con su liposolubilidad, que es el factor que determina su paso, más o menos rápido, a través de la barrera hematoencefálica y por lo tanto su efecto en el SNC. De los fármacos clasificados dentro de la familia de los barbitúricos, destacan los oxibarbitúricos, cuyo principal exponente es el pentobarbital y los tiobarbitúricos, cuyo principal representante es el tiopental.

En los estudios publicados en Estados Unidos, el barbitúrico más utilizado ha sido el pentobarbital mientras que en los europeos, el tiopental ha sido el fármaco más empleado. Las razones de esta variabilidad están en que en Europa, el pentobarbital no esta comercializado.

Hasta el momento, no se ha realizado ningún estudio controlado que compare la eficacia del pentobarbital y el tiopental en el control de la HIC. A nivel experimental sí hay trabajos que demuestran que sus mecanismos de acción difieren ${ }^{3,4,9,18,19}$. Por ello pensamos que es necesario un estudio que compare la eficacia de ambos fármacos en el control de la hipertensión intracraneal refractaria en pacientes con un TCE grave.

\section{Material y métodos}

El estudio se realizó en el Hospital Universitario Son Dureta (Palma de Mallorca), y fue aprobado por el Comité Ético de las Islas Baleares el 21 de marzo del 2002.

En todos los casos, se solicitó el consentimiento informado por escrito del familiar más cercano o del representante legal del paciente.

\section{Hipótesis y objetivos}

La hipótesis de trabajo fue que el tiopental puede ser más eficaz que el pentobarbital en el control de la PIC en pacientes con TCE grave, y que la incidencia de efectos secundarios no es superior con el tratamiento con tiopental.

El objetivo primario fue por lo tanto comparar la eficacia del pentobarbital y el tiopental en el control de la HIC refractaria en pacientes con TCE. Los objetivos secundarios fueron comparar la incidencia de efectos secundarios, principalmente la hipotensión arterial grave, que se definió como la presencia de una tensión arterial media inferior a $80 \mathrm{mmHg}$, en algún momento del coma barbitúrico.

\section{Criterios de inclusión}

En el estudio de carácter prospectivo se incluyeron todos los pacientes que ingresaron un nuestro centro entre mayo del 2002 y octubre del 2003, con el diagnóstico de TCE grave (puntuación de la escala de coma de Glasgow postreanimación, no quirúrgica, inferior o igual a 8 puntos) 
y que presentaron HIC refractaria a las medidas de primer nivel según las Guías de la Brain Trauma Foundation¹. El concepto de HIC refractaria se definió de acuerdo con los siguientes criterios:1) PIC entre 20-29 mmHg durante 30 minutos, 2) PIC entre 30-39 mmHg durante al menos 15 minutos, 3) PIC superior a $40 \mathrm{mmHg}$ durante más de un minuto.

\section{Criterios de exclusión}

No se incluyeron en el estudio los pacientes con una edad inferior a 15 años o superior a 76 años, pacientes con una puntuación en la escala de Coma de Glasgow (GCS) de 3 puntos al ingreso y con exploración compatible con muerte encefálica, pacientes embarazadas, aquellos con alergia o intolerancia a los barbitúricos, pacientes con disfunción ventricular cardíaca grave con fracción de eyección inferior al 35\% y todos aquellos que presentaban algún proceso concomitante o previo al ingreso y que, a criterio del investigador, pudiera verse afectado negativamente por la participación del paciente en el estudio.

\section{Intervenciones (protocolo terapéutico general)}

A todos los pacientes con un TCE grave se les realizó al ingreso una tomografía craneal computarizada (TC) y se les categorizó de acuerdo con la clasificación propuesta por el "Traumatic Coma Data Bank"14. A todos los pacientes se les monitorizó la PIC mediante un catéter intraparenquimatoso tipo Camino (Integra Neurosciences, Plainsboro, NJ, USA) y también se monitorizó la saturación de oxígeno en el bulbo de la yugular de forma intermitente cada 6 horas. La monitorización sistémica de estos pacientes incluyó: tensión arterial invasiva, pulsioximetría y catéter de Swan Ganz.

Los objetivos generales del tratamiento en los pacientes con TCE grave fueron mantener una tensión arterial media superior a $90 \mathrm{mmHg}$, una $\mathrm{PIC}$ inferior a $20 \mathrm{mmHg}$ y una presión de perfusión cerebral superior a $70 \mathrm{mmHg}$.

Los aumentos de la PIC por encima de $20 \mathrm{mmHg}$ fueron tratados de acuerdo a un protocolo estandarizado que incluyó: sedación, relajación, drenaje ventricular (cuando el paciente era portador de ventriculostomía), manitol al $20 \%$, suero salino hipertónico e hiperventilación moderada ( $\mathrm{pCO}_{2}$ entre $30-35 \mathrm{mmHg}$ ). Este tratamiento estandarizado se puede considerar convencional y está recogido en las Guías de Práctica Clínica de la "Brain Trauma Foundation" como terapias de primer nivel ${ }^{1}$.

En aquellos casos en que el tratamiento convencional fracasó en controlar la HIC, se decidió incluir el paciente en el estudio. Previamente a la aleatorización del paciente, se exigió que el tratamiento médico fuese máximo. Además ,se exigió también la realización de una TC durante las 24 horas previas a la inclusión del paciente en el estudio, haber administrado un bolo de manitol de $0,7 \mathrm{gr} / \mathrm{kg}$ una hora antes de la aleatorización, o tener una osmolaridad plasmática medida mayor de $320 \mathrm{mOsm} / \mathrm{kg}$.

\section{Aleatorización}

El diseño del estudio fue prospectivo, de cohortes, aleatorizado y de intervención terapéutica, comparativo entre dos fármacos (tiopental versus pentobarbital). La aleatorización se realizó siguiendo una lista generada por ordenador en la que se intercalaron ambos fármacos. Dicha aleatorización fue realizada por el médico de guardia de UCI, una vez que el paciente cumplía los criterios de inclusión y no presentaba ningún criterio de exclusión. La recogida de datos y el seguimiento de los pacientes fueron realizados por la misma persona (J. Pérez Bárcena) que conocía que fármaco se había administrado.

\section{Enmascaramiento}

El estudio no fue ciego ya que no se pudo enmascarar el tratamiento porque el tiopental se presenta en forma liofilizado y el pentobarbital no.

Forma de administración de los fármacos y monitorización de sus efectos

El pentobarbital se administró mediante una dosis de carga $10 \mathrm{mg} / \mathrm{kg}$ en 30 minutos seguida de una perfusión continua en bomba de $5 \mathrm{mg} / \mathrm{kg} /$ hora durante 3 horas. A partir de este punto se continuó con una dosis de mantenimiento de $1 \mathrm{mg} / \mathrm{kg} / \mathrm{h}$.

El tiopental se administró mediante un bolus de 2 $\mathrm{mg} / \mathrm{kg}$, administrado durante 20 segundos. En caso de no descender la PIC por debajo del umbral de $20 \mathrm{mmHg}$, se podía administrar un segundo bolus de $3 \mathrm{mg} / \mathrm{kg}$, que posteriormente se podía repetir si la PIC persistía elevada, a dosis de $5 \mathrm{mg} / \mathrm{kg}$. El mantenimiento se realizó mediante una perfusión continua de tiopental a $3 \mathrm{mg} / \mathrm{kg} / \mathrm{h}$.

En aquellos casos en que, después de haber instaurado el tratamiento de mantenimiento con ambos fármacos, no se hubiera conseguido un descenso de la PIC por debajo de $20 \mathrm{mmHg}$, se podía aumentar la dosis de mantenimiento a razón de $1 \mathrm{mg} / \mathrm{kg} / \mathrm{h}$.

En todos los casos, la monitorización del coma barbitúrico se realizó a través de la medición de los niveles de los fármacos en sangre y mediante la práctica de un electroencefalograma (EEG) diario. En el EEG se buscó la existencia del patrón denominado de salvas de supresión. Los niveles de pentobarbital y tiopental se midieron mediante la técnica de cromatografía líquida de alta resolución con detección ultravioleta y preparación de la muestra con extracción en fase líquida,

\section{Criterios de eficacia}

Se definió que existió una adecuada respuesta al tratamiento si la PIC descendía por debajo de los $20 \mathrm{mmHg}$, manteniéndose por debajo de este umbral durante al menos 
48 horas.

Se consideró que el tratamiento fracasaba cuando la PIC era incontrolable. Para los propósitos de este protocolo se definió una PIC incontrolable como aquélla que a pesar del coma barbitúrico se mantenía en los siguiente umbrales: 1) PIC entre 21-35 mmHg durante cuatro horas, 2) PIC entre 36-40 $\mathrm{mmHg}$ durante una hora o 3) $\mathrm{PIC}>41 \mathrm{mmHg}$ durante cinco minutos.

\section{Retirada del fármaco}

Cuando la PIC se mantenía controlada $(<20 \mathrm{mmHg}$ durante 48 horas) la retirada del coma barbitúrico se realizaba de forma progresiva y escalonada, disminuyendo la dosis a razón del $50 \%$ cada 24 horas hasta suspender la perfusión.

\section{Tamaño muestral}

Aceptando un riesgo alfa de 0,02 y un riesgo beta de 0,2 en un contraste de hipótesis bilateral, se estimó que eran necesarios 47 pacientes en cada grupo para detectar una diferencia igual o superior al $30 \%$ en el control de la HIC. Para calcular el tamaño muestral se asumió que la proporción de respuesta terapéutica en el grupo de pentobarbital era del $50 \%$ sin pérdidas en el seguimiento.

\section{Análisis estadístico}

Las variables cuantitativas se expresan mediante su media y desviación estándar de la media (DE). Las variables cualitativas están expresadas mediante porcentajes y sus intervalos de confianza al 95\% (IC:95\%). Para la comparación entre variables cuantitativas se utilizó la prueba de la $\mathrm{t}$ de Student si la variable seguía una distribución normal. En caso de que su distribución no fuera compatible con la normalidad, se ha utilizó la prueba de la U de MannWhitney. Para la comparación entre variables cualitativas se utilizó la prueba de la $\gamma$ i cuadrado o el test exacto de Fisher. Se ha considerado significación estadística cuando la probabilidad de error alfa en los contrastes de hipótesis fue inferior o igual al $5 \%(\mathrm{P} \leq 0,05)$.

\section{Resultados}

Desde mayo del 2002 hasta octubre del 2003 ingresaron en la UCI del Hospital Universitario Son Dureta 136 pacientes con el diagnóstico de TCE. Veinticuatro de estos pacientes presentaron HIC refractaria a las medidas de primer nivel, de los cuales 20 se incluyeron en este estudio. De los otros cuatro casos con HIC refractaria, dos no se incluyeron porque no presentaban todos los criterios de inclusión, otro fue trasladado a otro hospital en las primeras 24 horas del ingreso y el otro paciente fue excluido por una aleatorización incorrecta.

Las características basales de los 20 pacientes inclui- dos, diez en cada grupo, se presentan en la Tabla 1. No hubo diferencias estadísticamente significativas respecto a los datos epidemiológicos, comorbilidades o lesiones asociadas al TCE.

El GCS de ingreso en el grupo tratado con tiopental fue de seis \pm tres puntos y en el grupo de pentobarbital de siete \pm dos puntos $(\mathrm{P}=0,4)$. Respecto a la $\mathrm{TC}$ de ingreso en la UCI, en el grupo del tiopental dos pacientes tenían una lesión difusa tipo II según la clasificación del "Traumatic Coma Data Bank"14; cuatro presentaban una lesión difusa tipo III; un caso de lesión difusa IV, y tres de lesión masa evacuada. En el grupo del pentobarbital: tres casos de lesión difusa tipo II; cinco de lesión difusa tipo III y dos de lesión expansiva evacuada.

Los resultados se presentan en la Tabla 2. En cinco, de los diez casos en el grupo que recibió tiopental, se controló la PIC, mientras que la PIC se controló en sólo dos casos tratados con pentobarbital $(\mathrm{P}=0,16)$. El número de administraciones de tratamiento hiperosmolar (manitol y suero salino hipertónico), durante el coma barbitúrico, fue similar en ambos grupos: $17 \pm 11$ en el grupo de tiopental y $14 \pm$ 12 en el de pentobarbital $(\mathrm{P}=0,6)$. La duración del coma barbitúrico con tiopental fue de $154 \pm 64$ horas y de $94 \pm$ 69 horas con pentobarbital $(\mathrm{P}=0,06)$. Hubo cinco casos de rebote de la PIC con tiopental y cuatro con pentobarbital $(\mathrm{P}=0,65)$.

Dos de los diez pacientes tratados con tiopental llevaban soporte inotrópico por inestabilidad hemodinámica antes de iniciarse el coma barbitúrico, mientras que esto no ocurrió en ningún caso en el grupo tratado con pentobarbital $(\mathrm{P}=0,13)$.

Durante el coma barbitúrico se inició el soporte inotrópico en dos casos de tiopental y en uno de pentobarbital $(\mathrm{P}=0,5)$. La dosis máxima de noradrenalina en el grupo de tiopental fue de $2,3 \mu \mathrm{c} / \mathrm{kg} / \mathrm{min}$, y en el de pentobarbital fue de $2,5 \mu \mathrm{c} / \mathrm{kg} / \mathrm{min}$. La dosis media de noradrenalina con tiopental fue de $0,8 \mu \mathrm{c} / \mathrm{kg} / \mathrm{min}$ y de $1 \mu \mathrm{c} / \mathrm{kg} / \mathrm{min}$ en el grupo de pentobarbital.

En ambos grupos, nueve pacientes presentaron una tensión arterial media inferior a $80 \mathrm{mmHg}$ en algún momento del coma barbitúrico. Todos los pacientes tratados con pentobarbital tuvieron una presión de perfusión cerebral inferior a $60 \mathrm{mmHg}$ en alguna ocasión; y ocho pacientes del grupo de tiopental $(\mathrm{P}=0,13)$. En un solo caso en cada grupo se detectó una saturación de oxígeno en el bulbo de la yugular inferior al 55\% .

Todos los pacientes estuvieron monitorizados con un catéter de Swan Ganz. En líneas generales, los pacientes del grupo de pentobarbital tuvieron una presión de enclavamiento en la arteria pulmonar y un gasto cardíaco mayor, y unas resistencias vasculares sistémicas menores, sobre todo durante el primer y segundo día del coma barbitúrico. No hubo diferencias entre ambos grupos res- 
Tabla 1

Datos epidemiológicos, comorbilidades y lesiones asociadas

\begin{tabular}{|l|c|c|c|}
\hline & $\begin{array}{c}\text { Tiopental } \\
(\mathbf{n = 1 0 )}\end{array}$ & $\begin{array}{c}\text { Pentobarbital } \\
(\mathbf{n = 1 0 )}\end{array}$ & P \\
\hline Datos epidemiológicos & & & $34 \pm 14$ \\
\hline Edad (años) (media \pm DE) & $32 \pm 19$ & 9 & 0,7 \\
\hline Sexo (varón) & 7 & $80 \pm 9$ & 0,2 \\
\hline Peso (Kg) & $72 \pm 14$ & $44 \pm 10$ & 0,7 \\
\hline SAPS II & $46 \pm 16$ & $29 \pm 17$ & 0,2 \\
\hline APACHE II & $21 \pm 6$ & $57 \pm 21$ & 0,4 \\
\hline APACHE III & $67 \pm 29$ & 30,6 & 0,6 \\
\hline ISS & 28,5 & & 0,16 \\
\hline Lesiones asociadas & & 2 & 0,06 \\
\hline Traumatismo torácico & 5 & 0 & 0,6 \\
\hline Traumatismo abdominal & 3 & 3 & - \\
\hline Trauma extremidades & 4 & 0 & \\
\hline Traumatismo vertebral & 0 & & \\
\hline
\end{tabular}

SAPS II: Simplified Acute Physiology Score. APACHE II; APACHE III: Acute Physiology and Chronic Health Evaluation. ISS: índices de gravedad específicos para pacientes traumáticos: Injury Severity Score

Tabla 2

Resultados: control de la presión intracraneal y supervivencia

\begin{tabular}{|l|c|c|c|}
\hline & $\begin{array}{c}\text { Tiopental } \\
(\mathbf{n = 1 0}\end{array}$ & $\begin{array}{c}\text { Pentobarbital } \\
(\mathbf{n = 1 0})\end{array}$ & P \\
\hline Control de la PIC & 5 & 2 & 0,16 \\
\hline Necesidad tratamiento hiperosmolar (n ${ }^{\circ}$ de veces) & $17 \pm 11$ & $14 \pm 12$ & 0,6 \\
\hline Éxitus hospital & 5 & 8 & 0,16 \\
\hline Éxitus 6 meses & 5 & 8 & 0,16 \\
\hline
\end{tabular}

PIC: presión intracraneal Necesidad de tratamiento hiperosmolar: número de administraciones de manitol y/o suero salino hipertónico durante el coma barbitúrico.

pecto al valor de la temperatura central, recogida a través del catéter de Swan Ganz. La temperatura en ambos grupos se mantuvo entre 34 y $35^{\circ} \mathrm{C}$. (datos no presentados).

No hubo diferencias significativas respecto a la presencia de efectos adversos (Tabla 3 ) salvo en la presencia de fracaso hepático, que se definió como la presencia de un valor de bilirrubina total superior a $3 \mathrm{mg} / \mathrm{dL}$ y/o una GOT o GPT superior a $80 \mathrm{U} / \mathrm{L}$.

Al alta hospitalaria cinco pacientes del grupo del tiopental habían fallecido y ocho en el grupo del pentobarbital $(\mathrm{P}=0,16)$. A los seis meses se mantuvo esta supervivencia.

\section{Discusión}

Los resultados preliminares de este estudio indican que el tiopental podría ser más eficaz que el pentobarbital en el control de la HIC refractaria, aunque estos resultados se deben interpretar con cautela dado nuestro reducido tamaño muestral y porque el estudio fue diseñado $\sin$ enmascaramiento.

El coma barbitúrico es actualmente la única terapia de la que existe evidencia clase II, según las Guías de Práctica Clínica de la "Brain Trauma Foundation", para el tratamiento de la HIC refractaria a las medidas de primer nivel ${ }^{2}$. Este hecho hace que sea probablemente la medida 
Tabla 3

Efectos secundarios durante el coma barbitúrico

\begin{tabular}{|l|c|c|c|}
\hline & $\begin{array}{c}\text { Tiopental } \\
(\mathbf{n = 1 0 )}\end{array}$ & $\begin{array}{c}\text { Pentobarbital } \\
(\mathbf{n = 1 0})\end{array}$ & $\mathbf{P}$ \\
\hline TAM<80 mmHg & 9 & 9 & 0,6 \\
\hline Infección respiratoria & 8 & 7 & 0,13 \\
\hline Infección urinaria & 0 & 0 & 0,3 \\
\hline Hemocultivos positivos & 2 & 2 & 0,3 \\
\hline Infección catéter PIC & 4 & 0 & 0,87 \\
\hline Infección SNC (LCR) & 1 & 3 & 0,03 \\
\hline Fracaso renal & 3 & 0 & 0,13 \\
\hline Fracaso hepático & 4 & 0 & 0 \\
\hline Fracaso hematológico & 2 & & \\
\hline
\end{tabular}

TAM<80 mmHg: detección en algún momento del coma barbitúrico de una cifra de tensión arterial media inferior a $80 \mathrm{mmHg}$. SNC: sistema nervioso central. LCR: líquido cefalorraquídeo. Fracaso renal: Creatinina $>3 \mathrm{mg} / \mathrm{dL}$, y/o requerimiento de diálisis y/o hemodiafiltración veno vena continua. Fracaso hepático: valor de bilirrubina total superior a $3 \mathrm{mg} / \mathrm{dL}$; o un valor de las transaminasas hepáticas superior a 80 U/L. Fracaso hematológico: plaquetopenia inferior a 50000 o presencia de criterios de coagulación intravascular diseminada.

Tabla 4

Características farmacocinéticas del tiopental y pentobarbital

\begin{tabular}{|l|c|c|}
\hline & Tiopental & Pentobarbital \\
\hline Unión a proteínas (\%) & $60,5-96,7$ & 5 \\
\hline Volumen de distribución $(\mathrm{L} / \mathrm{kg})$ & $2,2-3,2$ & $0,65-0,95$ \\
\hline Aclaración $(\mathrm{ml} / \mathrm{h} / \mathrm{kg})$ & 0,074 & 0,72 \\
\hline Vida media (horas) & $6-46$ & $15-48$ \\
\hline Metabolitos activos & Pentobarbital & No \\
\hline
\end{tabular}

de segundo nivel más utilizada, con una frecuencia en su empleo que oscila en los diferentes estudios entre 13 y $56 \%{ }^{7,8,11}$. De aquí la importancia de comprobar la eficacia de los diferentes barbitúricos de que disponemos para controlar la PIC refractaria a medidas de primer nivel.

\section{Diferencias entre los oxibarbitúricos y tiobarbitúricos}

Las características farmacocinéticas (Tabla 4) del tiopental y pentobarbital son distintas ya que su unión a proteínas, volumen de distribución y aclaramiento siguen distintos patrones ${ }^{21}$. Sin embargo, el parámetro farmacológico fundamental, que es su vida media (tiopental: 6-46 horas; pentobarbital 15-48 horas), apenas varía de uno a otro grupo, y por tanto no parece que estas diferencias farmacocinéticas puedan tener repercusiones clínicas.

Una diferencia entre ambos grupos de barbitúricos es la presencia de metabolitos activos. El tiopental tiene cinco metabolitos, de los cuales cuatro son inactivos y uno es activo: el pentobarbital (pentobarbitona). Por tanto, el pentobarbital es un metabolito activo del tiopental. Este hecho, junto con la existencia de una gran variabilidad intra e interindividual en el metabolismo de estos fármacos, debido a la existencia de fenómenos de inducción enzimática del citocromo p450 hepático, hacen que exista una escasa correlación entre las concentraciones séricas y beneficios terapéuticos, y que por tanto sea más recomendable la monitorización de este tratamiento mediante el electroencefalograma.

A nivel experimental se han realizado varios trabajos que han comparado ambos fármacos. Hatano et $\mathrm{al}^{9}$, en un estudio realizado con perros, llegaron a la conclusión de que los tiobarbitúricos provocan vasoconstricción cerebral, lo cual podría favorecer la redistribución del flujo sanguíneo cerebral. Cole et $\mathrm{al}^{4}$ demostraron que el tiopental 
reduce el tamaño del área isquémica más que el pentobarbital, a pesar de que con ambos fármacos se conseguía el patrón electroencefalográfico de salvas de supresión. Shibuta ${ }^{18}$ observó que el tiopental, pero no el pentobarbital, era capaz de atenuar el daño citotóxico mediado por el óxido nítrico. Almaas et al. ${ }^{3}$ observaron que los diferentes barbitúricos tienen distintos efectos neuroprotectores frente a la deprivación de oxígeno y glucosa, en un modelo con cultivos de neuronas humanas. El tiopental ejerció un efecto neuroprotector a todas las dosis estudiadas, mientras que el pentobarbital sólo a dosis elevadas. Por último, Smith et $\mathrm{al}^{19}$ demostraron en un estudio in vitro, que mientras el tiopental causaba una inhibición del $96 \%$ de la peroxidación lipídica, el pentobarbital apenas tenía efecto alguno.

Todos estos estudios demuestran que todos los barbitúricos no son iguales y por tanto su capacidad neuroprotectora puede diferir, por lo que a pesar de las limitaciones metodológicas de nuestro estudio, pensamos que está justificado continuarlo ${ }^{6}$.

\section{Efectos secundarios del coma barbitúrico}

El efecto secundario detectado con más frecuencia fue, como era de esperar, la hipotensión arterial, que ocurrió en nueve pacientes en cada grupo. Esta incidencia es quizá mayor que la de estudios previos ${ }^{7}$, pero pensamos que es debida a la definición de hipotensión empleada, aunque también puede verse influída por el tamaño de la muestra. Hay que decir que todos los enfermos estaban monitorizados con un catéter de Swan Ganz y los episodios de hipotensión arterial se trataron de forma enérgica con fluidoterapia y drogas vasoactivas. En líneas generales, los pacientes tratados con tiopental tuvieron más efectos secundarios que los que recibieron pentobarbital, quizás porque la duración del coma barbitúrico con tiopental fue mayor que con pentobarbital. De hecho, un efecto secundario que ocurrió en el grupo del tiopental, pero no en el de pentobarbital, fue la aparición de un fracaso hepático, definido por la detección de un valor de bilirrubina total superior a $3 \mathrm{mg} / \mathrm{dL}$ o un valor de la GOT y GPT superior a 80 U/L. Sin embargo, la detección de una hiperbilirrubinemia o una elevación de las transaminasas no es infrecuente en los pacientes ingresados en la UCI, y suele ser de origen multifactorial. Además, en el grupo del tiopental hubo tres pacientes con un traumatismo abdominal, mientras que no hubo ninguno en el grupo tratado con pentobarbital, lo que también podría justificar estos hallazgos.

\section{Conclusión}

Los resultados preliminares de este estudio indican que el tiopental podría ser más eficaz que el pentobarbital en el control de la hipertensión intracraneal refractaria a medidas de primer nivel, según las Guías de Práctica Clínica de la
"Brain Trauma Foundation". Dichos resultados confirman la evidencia experimental que indica que los mecanismos neuroprotectores de ambos fármacos son distintos y justifican el continuar aumentando el tamaño muestral de nuestro estudio para poder definir la eficacia de ambos fármacos.

Este trabajo está financiado por la beca Fondo de Investigación Sanitaria (FIS PI020642) concedida al Dr J Pérez Bárcena

\section{Bibliografía}

1. The Brain Trauma Foundation. The American Association of Neurological Surgeons. The Joint Section on Neurotrauma and Critical Care. Critical pathway for the treatment of established intracranial hypertension. J. Neurotrauma 2000; 17: 537-538.

2. The Brain Trauma Foundation. The American Association of Neurological Surgeons. The Joint Section on Neurotrauma and Critical Care. Use of barbiturates in the control of intracranial hypertension. J. Neurotrauma 2000; 17 : 527-530.

3. Almaas, R., Saugstad, O.D., Pleasure, D., et al.: Effect of barbiturates on hydroxyl radicals, lipid peroxidation, and hypoxic cell death in human NT2-N neurons. Anesthesiology 2000; 92: 764-774.

4. Cole, D.J., Cross, L.M., Drummond, J.C., et al.: Thiopentone and methohexital, but not pentobarbitone, reduce early focal cerebral ischemic injury in rats. Can. J. Anaesth. 2001; 48: 807-814.

5. Demopoulos, H.B., Flamm, E.S., Pietronigro, D.D., et al.: The free radical pathology and the microcirculation in the major central nervous system disorders. Acta Physiol Scand. 1980; Suppl 492: 91-119.

6. Drummond, J.C., Patel, P.M., Cole, D.J.: Cerebral protection: are all barbiturates created equal? Anesthesiology 1996; 85: 1504-1505.

7. Eisenberg, H.M., Frankowski, R.F., Contant, C.F., et al.: High-dose barbiturate control of elevated intracranial pressure in patients with severe head injury. J. Neurosurg. 1988; 69: 15-23.

8. Ghajar, J., Hariri, R.J., Narayan, R.K., et al.: Survey of critical care management of comatose, head-injured patients in the United States. Crit Care Med. 1995; 23: 560-567.

9. Hatano, Y., Nakamura, K., Moriyama, S., et al.: The contractile responses of isolated dog cerebral and extracerebral arteries to oxybarbiturates and thiobarbiturates. Anesthesiology 1998; 71: 80-86.

10. Horsley, J.S.: The intracranial pressure during barbital narcosis. Lancet 1937; 1: 141-143.

11. Jeevaratnam, D.R., Menon, D.K.: Survey of intensive care of severely head injured patients in the United Kingdom. BMJ 1996; 312: 944-947. 
12. Kassell, N.F., Hitchon, P.W., Gerk, M.K., et al.: Alterations in cerebral blood flow, oxygen metabolism, and electrical activity produced by high dose sodium thiopental. Neurosurgery $1980 ; 7$ : 598-603.

13. Marshall, L.F., Bruce, D.A., Bruno, L., et al.: Role of intracranial pressure monitoring and barbiturate therapy in malignant intracranial hypertension. Case report. J. Neurosurg. 1977; 47: 481-484.

14. Marshall, L.F., Marshall, S.B., Klauber, M.R., et al.: A new classification of head injury based on computerized tomography. J Neurosurg 1991; 75: S14-S20.

15. Marshall, L.F., Shapiro, H.M.: Barbiturate control of intracranial hypertension in head injury and other conditions: iatrogenic coma. Acta Neurol.Scand. 1977; Suppl 64: 156157.

16. Marshall, L.F., Smith, R.W., Shapiro, H.M.: The outcome with aggressive treatment in severe head injuries. Part I: the significance of intracranial pressure monitoring. J. Neurosurg. 1979; 50: 20-25.

17. Rockoff, M.A., Marshall, L.F., Shapiro, H.M.: Highdose barbiturate therapy in humans: a clinical review of 60 patients. Ann.Neurol. 1979; 6: 194-199.

18. Shibuta, S., Kosaka, J., Mashimo, T., et al.: Nitric

\section{Comentario al trabajo Comparación de la eficacia del pentobarbital y el tiopental en el control de la hipertensión intracraneal refractaria. Resultados preliminares en una serie de 20 pacientes de Pérez Bárcena y cols.}

Pérez-Bárcena y cols., presentan los resultados preliminares de un estudio prospectivo y aleatorizado en el que se compara la eficacia de dos tipos de barbitúricos en el control de la hipertensión intracraneal (HIC) refractaria al tratamiento médico recomendado por las guías de práctica clínica de la Brain Trauma Foundation (BTF) ${ }^{1}$. La presentación del diseño, desarrollo y resultados de este estudio siguen las directrices y recomendaciones del método "CONSORT" (Consolidated Standards Of Reporting Trials) en su última revisión ${ }^{2}$. El paradigma CONSORT constituye una herramienta o estrategia metodológica diseñada en la década de los noventa y dirigida a mejorar la calidad de los estudios con distribución aleatoria de los pacientes en los que se compara la eficacia de dos fármacos o medidas terapéuticas. Esta estrategia metodológica implica el seguimiento de un guión o checklist de 22 ítems, en el que se incluye una determinada presentación de los diferentes apartados del artículo. Su uso incrementa la transparencia del estudio, al mismo tiempo que ayuda a los revisores y lectores a analizar la idoneidad e interpretación de los resultados.

La administración de barbitúricos en el tratamiento de los pacientes que han presentado un traumatismo oxide-induced cytotoxicity attenuation by thiopentone sodium but not pentobarbitone sodium in primary brain cultures. Br. J. Pharmacol. 1998; 124: 804-810.

19. Smith, D.S., Rehncrona, S., Siesjo, B.K.: Inhibitory effects of different barbiturates on lipid peroxidation in brain tissue in vitro: comparison with the effects of promethazine and chlorpromazine. Anesthesiology. 1980; 53: 186-194.

20. Steen, P.A., Michenfelder, J.D.: Cerebral protection with barbiturates: relation to anesthetic effect. Stroke.1978; 9: 140-142.

21. Steen, P.A., Michenfelder, J.D.: Mechanisms of barbiturate protection. Anesthesiology. 1980; 53: 183-185.

Pérez-Bárcena, J.; Barceló, B.; Homar, J.; Abadal, J.M.; Molina, F.J.; de la Peña, A.; Sahuquillo, J.; Ibáñez, J.: Comparación de la eficacia del pentobarbital y el tiopental en el control de la hipertensión intracraneal refractaria. Resultados preliminares en una serie de 20 pacientes. Neurocirugía 2005; 16: 5-13.

Correspondencia postal: Jon Pérez Bárcena. Servicio de Medicina Intensiva. Hospital Son Dureta. Andrea Doria 55. 07014. Palma Mallorca

craneoencefálico (TCE) constituye una maniobra frecuente en el contexto de estos pacientes. Sin embargo, a pesar de las recomendaciones de la BTF, existen todavía controversias en el uso de estos fármacos. En su última revisión, la fundación Cochrane reconoce la eficacia de los barbitúricos en el control de la presión intracraneal (PIC), aunque enfatiza que, hasta el momento, no se ha conseguido objetivar un claro beneficio en el resultado final de los pacientes que presentan una HIC refractaria a las medidas terapéuticas de primer nivel ${ }^{3}$. Esto puede deberse a la escasa literatura que existe en el uso clínico de los barbitúricos, al tamaño de la muestra de estudios que han sido considerados clásicamente como los más relevantes y al hecho de que todavía no se conozcan plenamente los mecanismos de acción de estos fármacos. El presente estudio ha sido correctamente diseñado y estructurado. Los resultados que los autores presentan podrían ser relevantes en el tratamiento de los pacientes neurotraumáticos, si estos datos preliminares se confirmaran en una serie más amplia. El reducido tamaño de la muestra es el principal inconveniente del estudio.

El mecanismo de acción aceptado como básico de los barbitúricos es la reducción del metabolismo cerebral funcional, que resulta de la disminución de la excitabilidad 
neuronal por su acción agonista de los receptores GABA. Dado el acoplamiento que existe entre metabolismo y flujo sanguíneo cerebral, a la disminución de las necesidades metabólicas le sigue una disminución del flujo, y por tanto, del volumen sanguíneo cerebral, lo que condiciona una reducción de la PIC. Su efecto máximo se consigue cuando el paciente presenta un patrón electroencefalográfico de "salvas de supresión". A partir de este punto, aumentar las dosis de barbitúricos sólo incrementa los no despreciables efectos secundarios asociados a estos fármacos. Algunos autores han propuesto mecanismos de acción adicionales de los barbitúricos en el descenso de la PIC, como es su potencial efecto vasoconstrictor cerebral ${ }^{4}$. Sin embargo, estos hallazgos no han sido confirmados por otros autores $^{5}$. A nivel sistémico, los barbitúricos reducen de forma importante las resistencias vasculares, por lo que uno de sus efectos secundarios más importantes es la inestabilidad hemodinámica y las caídas de la tensión arterial que conllevan descensos de la presión de perfusión cerebral. Posiblemente, el descenso de la tensión arterial en un grupo de pacientes muy graves, con HIC refractaria y que, con toda probabilidad, no autorregulan, contribuye al descenso de la PIC. Otro factor a considerar, y que también han constatado los autores del presente estudio, es que la administración de barbitúricos desciende la temperatura corporal, siendo bien conocido que la hipotermia también disminuye de forma importante la PIC.

En el tratamiento de la HIC refractaria pueden emplearse diferentes tipos de barbitúricos. Hasta hace poco tiempo en España sólo se podía utilizar de forma rutinaria el tiopental. El pentobarbital, utilizado de forma habitual en otros países, constituye el metabolito activo del tiopental $\mathrm{y}$ ofrece la ventaja adicional de una mayor facilidad en la monitorización de los niveles plasmáticos del fármaco. No obstante, el tiopental genera otros metabolitos que se han relacionado con un efecto neuroprotector adicional, derivado de su potencial acción en diversos puntos de las cascadas metabólicas anómalas y procesos inflamatorios que presentan los pacientes con un TCE grave. Sin embargo, los expertos de la $\mathrm{BTF}^{6}$ reconocen que, en el momento actual, no se dispone de la suficiente información para recomendar el uso de un determinado barbitúrico; de aquí la importancia del presente estudio, a pesar de que en el momento actual sólo constituya una fase preliminar de lo que pretenden realizar los autores.

\section{Bibliografía}

1. The Brain Trauma Foundation. The American Association of Neurological Surgeons. The Joint Section on Neurotrauma and Critical Care. Management and prognosis of severe traumatic brain injury. Part 1: Guidelines for the management of severe traumatic brain injury. J. Neurotrauma. 2000; 17: 451-627.

2. Moher, D., Schulz, K.F., Altman, D.G., et al.: The CONSORT statement: revised recommendations for improving the quality of reports of parallel-group randomised trials. Lancet. 2001; 357: 1191-1194.

3. Roberts, I.: Barbiturates for acute traumatic brain injury. Cochrane Database Syst Rev. 2000; (2):CD000033.

4. Hatano, Y., Nakamura, K., Moriyama, S., Mori, K., Toda, N.: The contractile responses of isolated dog cerebral and extracerebral arteries to oxybarbiturates and thiobarbiturates. Anesthesiology. 1989; 71:80-86.

5. Marin, J., Lobato, R.D., Rico, M.L., Salaices, M., Benitez, J.: Effect of pentobarbital on the reactivity of isolated human cerebral arteries. J. Neurosurg. 1981; 54: 521-524.

6. Adelson, P.D, Bratton, S.L., Carney, N.S. et al.: Guidelines for the acute medical management of severe traumatic brain injury in infants, children, and adolescents. Chapter 13. The use of barbiturates in the control of intracranial hypertension in severe pediatric traumatic brain injury. Pediatr.Crit.Care. 2003; 4; S49-S51.
M.A. Poca Barcelona 